\title{
Multi-objective model selection algorithm for online sequential ultimate learning machine
}

\author{
Xinyuan $\mathrm{Jin}^{2}$, Tao $\mathrm{He}^{3^{*}}$ and Yezhi Lin ${ }^{1,4^{*}}$
}

\begin{abstract}
In order to improve the channel equalization, improve the control quality, and reduce the error of the target output of the online sequential learning machine, a multi-objective model selection algorithm is proposed based on feedback compensation and adaptive equalization control. The channel equalization model of online sequential ultimate learning machine is constructed. The sensor fusion information of online sequential limit learning machine is selected adaptively by multi-objective combined control, and the multi-objective combined control is carried out by using matched filtering method. Combined with feedback compensation and adaptive equalization control method, the classification selection and equalization of network multi-objective models are realized. The simulation results show that the algorithm has good accuracy in classifying and selecting multi-objective models of online sequential LLM, the adaptive equalization performance of the channel is better, and the error of LLM control is low.
\end{abstract}

Keywords: Online sequential extreme learning machine, Multi-objective model, Classification selection, Equilibrium

\section{Introduction}

With the development of network information technology, using network control to transmit data becomes a necessary means for people to transmit data and exchange information. An online sequential extreme learning machine is constructed to realize low frequency, middle frequency, and high frequency. VHF and UHF signals interact with information and improve the quality of data transmission through channel equalization and modulation and demodulation. The traditional network is a platform for information transmission, and receiving and sharing users can connect various kinds of rich information together, so as to realize the information sharing between different regions. Cognitive network is a new advanced communication technology in recent years. The technology of perception, learning, and reconfiguration is adopted comprehensively, so it has important application value [1]. Cognitive network is a

\footnotetext{
*Correspondence: vdd0na@163.com; 178221103@qq.com

${ }^{3}$ Department of Electrical and Electronic Engineering, Wenzhou Vocational \&

Technical College, Wenzhou 325035, People's Republic of China

${ }^{1}$ The First Affiliated Hospital of Wenzhou Medical University, Wenzhou

325000, People's Republic of China

Full list of author information is available at the end of the article
}

network with cognitive process. It can understand and learn by itself by perceiving the current network environment. Then the internal configuration is adjusted to adapt to the change of the external network according to the understanding and the learning knowledge [2]. It has a certain adaptive ability. That is, it can continuously learn and accumulate relevant knowledge in the process of dynamic adaptation, and based on it, it can adjust, decide, and reconfigure the network [3]. In order to improve the data configuration and clustering ability of the network, it is necessary to use the ultimate learning machine to optimize the target combination control, to improve the network transmission control ability and to realize the optimal selection of the multiobjective model of the ultimate learning machine.

Extreme learning machine (ELM) algorithm is proposed by Huang based on a single-hidden layer feedforward neural network (SLFN) algorithm. Because of its strong mathematical background, fast learning speed, ability to approach complex nonlinear functions, and high generalization ability, ELM algorithm has become a widely used machine learning technology. It has been successfully applied to many fields, 
such as pattern recognition, data mining, and so on [4]. Different from the traditional neural network algorithm based on gradient descent and error back propagation, it is necessary to iterate and determine the optimal solution of all parameters several times during the training process, which results in a large amount of computation [5]. The slow learning speed of ELM algorithm is taken based on the fact that the learning ability of SLFN is only related to the number of hidden layer nodes but independent of the weight of the input layer. Therefore, in the process of ELM learning, a large number of artificial network parameters are not required, and the learning speed of the algorithm is significantly higher than that of the traditional neural network learning algorithm, because the ELM learning algorithm considers all training samples equally. Each positive class sample and negative class sample in the dataset have the same effect on the formation of the classification surface, while the negative class accumulates as all the samples of most classes, and finally has a great influence on the formation of the classification surface [6].

In order to solve the above problems, this paper proposes a multi-objective model selection algorithm based on feedback compensation and adaptive equalization control. Firstly, the channel equalization model of online sequential ultimate learning machine is constructed. The sensor fusion information of online sequential limit learning machine is selected adaptively by multi-objective combined control, and the multi-objective combined control is carried out by using matched filtering method. Combined with feedback compensation and adaptive equalization control method, the classification selection and equalization of network multi-objective models are realized. Finally, the performance test is carried out through the simulation experiment, which shows the superior performance of this method in improving the ability of selecting the multi-objective model of the ultimate learning machine.

\section{Methods}

\subsection{Channel characteristics of online sequential extreme} learning machine

In order to optimize the classification and selection of multi-objective models of molecular exchange networks and improve the network performance and data transmission capability, the channel model of online sequential extreme learning machine should be constructed first. The channel of online sequential extreme learning machine is a limited bandwidth [7]. The tap delay line model of the channel is a wideband channel transmission model. Let the time delay of each multipath reach be an integer multiple of the width of symbol. The channel impulse response of the online sequential extreme learning machine can be expressed as follows:

$$
h(t)=\sum_{i} a_{i}(t) e^{j \theta_{i}(t)} \delta\left(t-i T_{S}\right)
$$

The wideband propagation characteristics of data transmission in online sequential extreme learning machine have a serious effect on the multipath propagation phenomenon in the actual network channel. Using the tap delay line model with tap interval MT/N to suppress multipath propagation will cause inter-symbol interference of transmitted signal, where $M$ and $N$ are integers and $M<N$. The received signal spectrum is equalized, and the channel characteristics in the online sequential learning machine are described as follows:

$$
\begin{aligned}
C_{T^{\prime}}(f) Y_{T^{\prime}}(f) & =C_{T^{\prime}}(f) \sum_{n} \\
& x\left(f-\frac{n}{T^{\prime}}\right) e^{j 2 \pi\left(f-\frac{n}{T^{\prime}}\right) \tau=C_{T^{\prime}}(f) X(f) e^{j 2 \pi f \tau}}
\end{aligned}
$$

where $X(f)$ is the bandwidth limited spectrum of channel impulse response of online sequential learning machine, $Y_{T^{\prime}}(f)$ is the spectrum of sampling signal, and $\tau_{0}$ is the time delay of data transmission by online sequential ultimate learning machine.

\subsection{Phase shift calculation of multi-objective model of online sequential extreme learning machine}

On the basis of the analysis of the channel characteristics of the online sequential limit learning machine, the phase shift of the multi-objective model of the online sequential limit learning machine is calculated, the

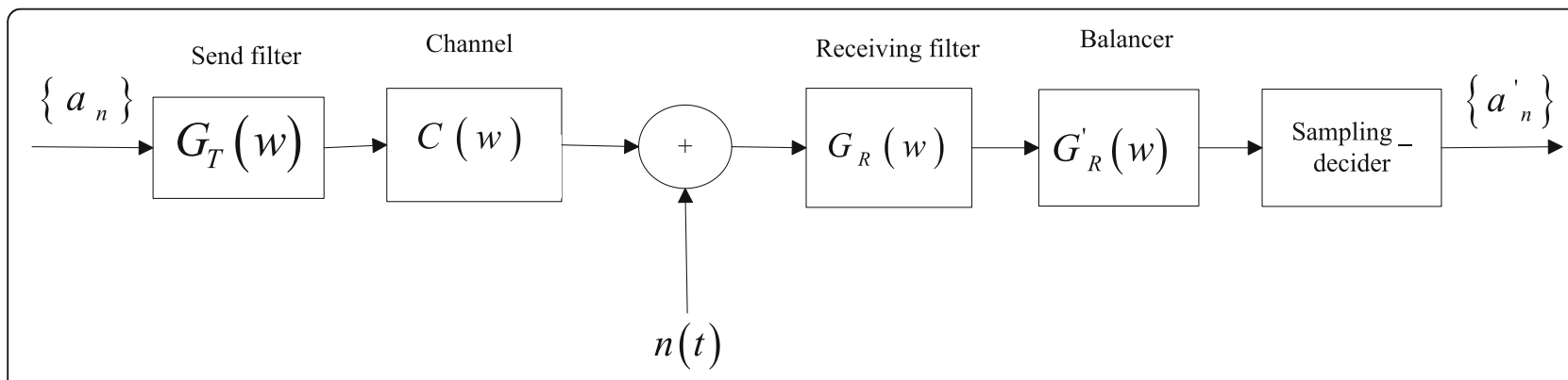

Fig. 1 Data transmission structure model of online sequential extreme learning machine 
migration is suppressed, the network channel model is constructed, and the channel average is improved [8]. On the assumption that the channel model expressed in the network is ideal, the amplitude equalization principle is used to modulate the channel. The online sequential extreme learning machine node is composed of $N=2 P$ elements, and the online sequential extreme learning machine data transmission system is based on the principle of amplitude equalization and channel modulation. The signal reception model is expressed as follows:

$$
x_{m}(t)=\sum_{i=1}^{I} s_{i}(t) e^{j \phi_{m i}}+n_{m}(t),-p+1 \leq m \leq p
$$

where $s_{i}(t)$ is the $i$ phase deflection information in the input component of the excessive path of the online sequential extreme learning machine node and $x_{m}(t)$ is the equalization transfer phase model of the receiving terminal of the online sequential ultimate learning machine, and the time delay of each multipath arrival is obtained [9]. The data transfer structure model of online sequential extreme learning machine is shown in Fig. 1.

Assuming that the length of LFM period in LLM is T, the multi-objective model is constructed by using multipath signal to realize time compression, and the data multi-objective model of online sequential LLM is obtained as follows:

$$
\begin{aligned}
& x(t)=\left[x_{-P+1}(t), x_{-P+2}(t), \ldots, x_{P}(t)\right]_{N \times 1}^{T} \\
& s(t)=\left[s_{1}(t), s_{2}(t), \ldots, s_{I}(t)\right]_{I \times 1}^{T}
\end{aligned}
$$

By adding phase shift of multipath component, the direct sequence spread spectrum component of online sequential extreme learning machine data transmission is obtained as follows:

$$
\boldsymbol{y}(k)=\boldsymbol{a}(k) \boldsymbol{h}(k)+\boldsymbol{n}(k)
$$

The multi-objective model of online sequential extreme learning machine is fixed compensated for shrinkage [10], and the filter is used to compensate the cost. The pulse response of equalizer compensation distortion is expressed as follows:

$$
J_{\mathrm{MDMMA}}=\rho \cdot E\left[\left(|z(k)|^{2}-R_{\mathrm{MDMMA}}(k)\right)^{2}\right]
$$

In order to reduce the steady-state error, the adaptive phase offset compensation is used to suppress the channel interference after receiving the training sequence. The iterative formula of interference suppression is obtained as follows:

$$
\boldsymbol{f}(k+1)=\boldsymbol{f}(k)-\mu \cdot \rho \cdot e_{\mathrm{MDMMA}}(k) \boldsymbol{y}^{*}(k)
$$

where

$$
e_{\mathrm{MDMMA}}(k)=z(k)\left[|z(k)|^{2}-R_{\mathrm{MDMMA}}(k)\right]
$$

Because the value of the iterative step size or the adaptive control factor of the equalizer has a great influence on the equalization algorithm, the value of $\rho$ is satisfied:

$$
\rho=\left\{\begin{array}{c}
1, \operatorname{sgn}\left(|z(k)|^{2}-R_{\mathrm{MDMMA}}(k)\right)=\operatorname{sgn}\left(|z(k)|^{2}-R\right) \\
0, \operatorname{sgn}\left(|z(k)|^{2}-R_{\mathrm{MDMMA}}(k)\right) \neq \operatorname{sgn}\left(|z(k)|^{2}-R\right)
\end{array}\right.
$$

The channel equalization model of online sequential ultimate learning machine is constructed. The sensor fusion information of online sequential limit learning machine is selected adaptively by multi-objective combined control [11].

\section{Improved algorithm for classification and selection of multi-objective models}

\subsection{Adaptive channel selection of ultimate learning} machine based on multi-objective combined control

On the basis of constructing the multi-objective model of online sequential limit learning machine, the optimization design of channel classification selection algorithm is carried out. An online sequential limit model based on feedback compensation and adaptive equalization control is proposed in this paper [12]. The classification and selection algorithm of learning machine multi-objective model is used to select the sensor fusion information of online sequential extreme learning machine by using multi-objective combined control. The matched filter structure model is used to optimize the channel transmission performance of the online sequential learning machine. The linear transverse equalization analysis is carried out by using the least mean square error (LMS) criterion [13]. It is obtained that the complete baud interval in the frequency band $p(t)$ of the ultimate learning machine is as follows:

$$
p_{r i}(t)=p(t) * h_{i}(t)+n_{p i}(t)
$$

The frequency response of the transverse equalization between the $i$ node in an online sequential extreme learning machine:

$$
C_{T^{\prime}}(f)=\sum_{k=-K}^{K} c_{k} e^{-j 2 \pi f k T^{\prime}}
$$

where $c_{k}$ is the normalized amplitude, $N$ is the sampling length of the recovery time frequency characteristic data, and $P$ is the spectrum characteristic of the received signal. In addition, the $T^{\prime}=\mathrm{MT} / N$ uses the information symbol detection decision, and the symbol width is $T_{a}, T_{a}=1 / R_{a}$, and the complex 


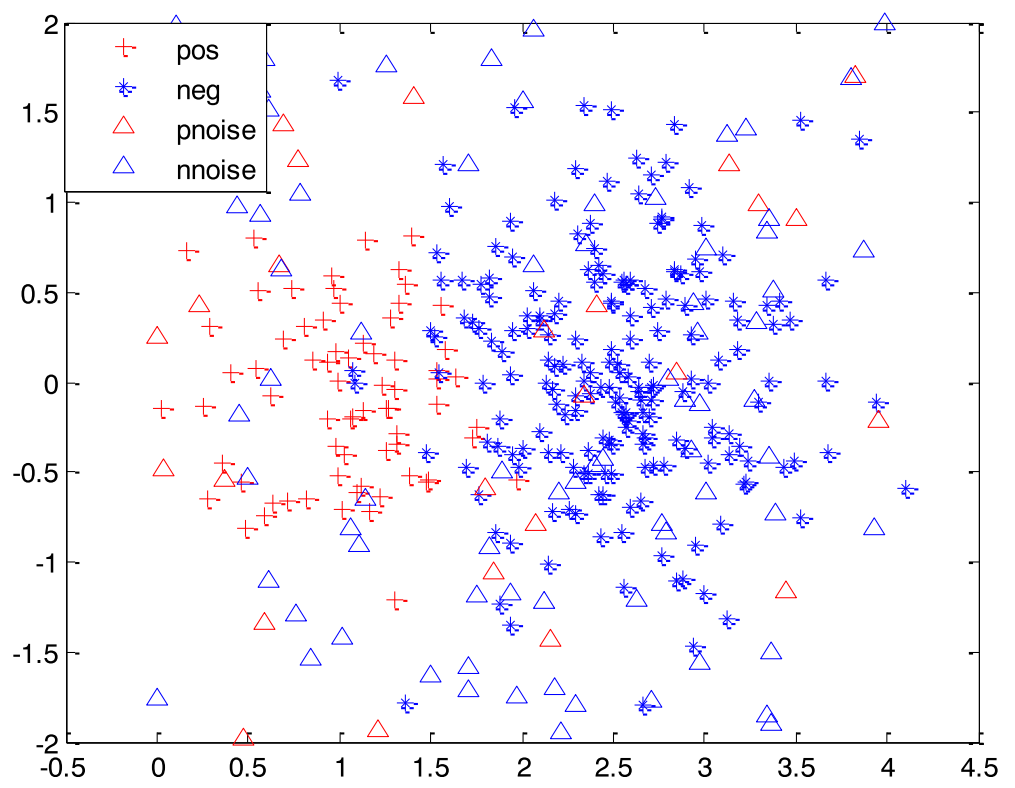

Fig. 2 Multi-objective model distribution of online sequential extreme learning machine

expression of the baseband envelope of the jamming signal is described as:

$$
\begin{aligned}
& \operatorname{sgn}\left(z_{R}^{2}(k)-R_{\text {MDMMA_R }}\right)=\operatorname{sgn}\left(z_{R}^{2}(k)-\hat{e}_{R}^{2}(k)\right) \\
& \operatorname{sgn}\left(z_{I}^{2}(k)-R_{\text {MDMMA_I }}\right)=\operatorname{sgn}\left(z_{I}^{2}(k)-\hat{e}_{I}^{2}(k)\right)
\end{aligned}
$$

Where, $\hat{e}_{R}^{2}(k)$ is the demodulation output sample of multi-objective model of online sequential extreme learning machine, $z_{R}^{2}(k)$ is an adjustable filter correction ratio, $z_{I}^{2}(k)$ is carrier frequency, and $\hat{e}_{I}^{2}(k)$ is over adjusted equalizer parameter.

According to the phase offset between each path and the direct path, the multi-objective combinatorial control is used to filter the channel by fuzzy adaptive [14], and the decision function of the multi-objective combined control is obtained as follows:

$$
\begin{aligned}
& \boldsymbol{f}_{F}(k+1)=\boldsymbol{f}_{F}(k)-\mu \cdot \nabla_{\boldsymbol{f}_{F}(k)} J_{\text {MMDMMA }} \\
& =\boldsymbol{f}_{F}(k)-\mu \cdot \nabla_{\boldsymbol{f}_{F}(k)}\left(J_{\text {MMDMMA_R }}+J_{\text {MMDMMA_I}}\right) \\
& =\boldsymbol{f}_{F}(k)-\mu_{F}\left[\rho(k) e_{\text {MDMMA }}(k)+(1-\rho(k)) e(k)\right] \boldsymbol{y}^{*}(k) \\
& =\boldsymbol{f}_{F}(k)-\mu_{F}\left[\rho(k)\left(e_{\text {MDMMA_R }}(k)+j e_{\text {MDMMA_I}}(k)\right)+\right. \\
& \left.\quad(1-\rho(k))\left(e_{R}(k)+j e_{I}(k)\right)\right] \boldsymbol{y}^{*}(k)
\end{aligned}
$$

The steady-state error of channel offset phase estimation in constellation circle is:

$$
e_{\mathrm{MDMMA}}(k)=z(k)\left[|z(k)|^{2}-R_{\mathrm{MDMMA}}(k)\right]
$$

The phase shift of multipath component is added, and the multi-objective combination control is used to filter the sensor fusion information of the online sequential limit learning machine [15]. The iterative transfer function of the adaptive filtering of phase information is obtained:

$$
d(t)=a(t) c(t)=\sum_{n=0}^{\infty} d_{n} g_{c}\left(t-n T_{c}\right)
$$

where

$$
d_{n}=\left\{\begin{array}{cc}
+1 & a_{n}=c_{n} \\
-1 & a_{n} \neq c_{n}
\end{array}(n-1) T_{c} \leq t \leq n T_{c}\right.
$$

Based on the multi-objective combined control method, the channel of online sequential extreme learning machine is selected adaptively. The decision error function of the channel equalization is obtained as follows:

$$
\begin{aligned}
\hat{e}(k)= & z_{R}(k)\left(\left|z_{R}(k)\right|^{2}-\hat{s}_{R}^{2}(k)\right)+j z_{I}(k) \\
& \times\left(\left|z_{I}(k)\right|^{2}-\hat{s}_{I}^{2}(k)\right)
\end{aligned}
$$

\subsection{Channel classification selection based on feedback compensation and adaptive equalization control}

The multi-objective model of LLM is classified and equalized through adaptive channel selection, feedback compensation, and adaptive equalization control. Based on the direct sequence spread spectrum principle, the constellation radius of the online sequential extreme learning machine is obtained as $R_{\text {MDMMA }_{-}(i=1, \cdots, N) \text {, and the QAM modulation }}$ $R_{M D M M A}(k)$ is used for Doppler expansion: 


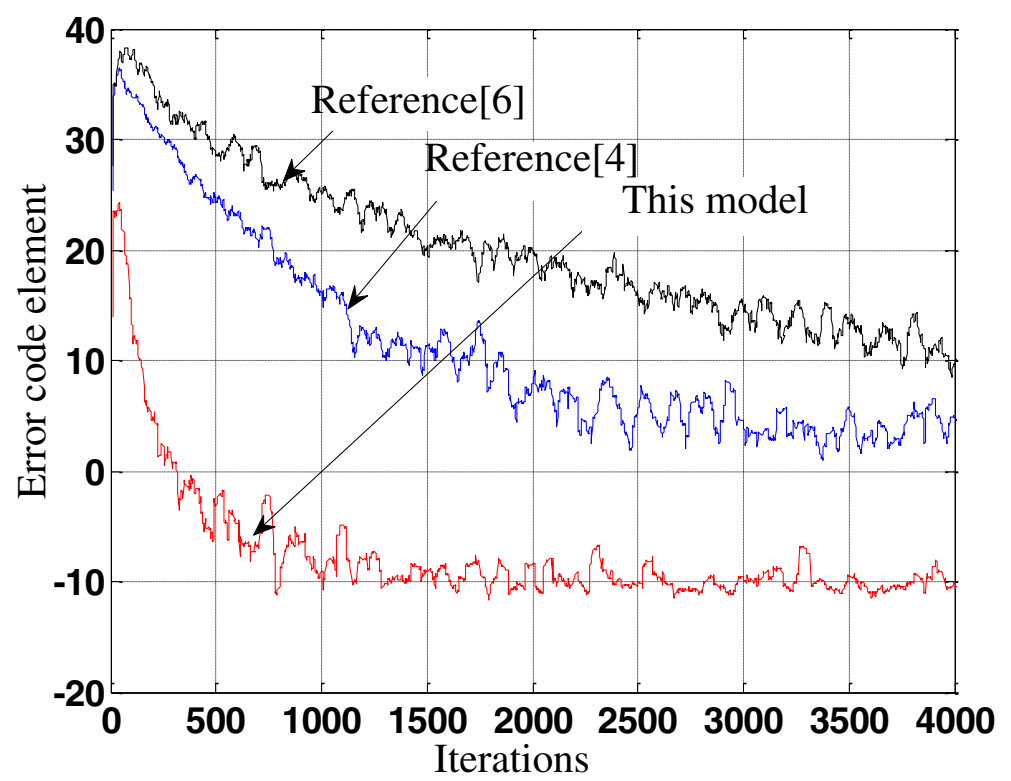

Fig. 3 Error code comparison of the output of online sequential extreme learning machine

$$
\begin{aligned}
& a b s\left[|z(k)|^{2}-R(k)^{\mathrm{MDMMA}}\right] \\
& \quad=\min _{i} a b s\left[|z(k)|^{2}-R_{\text {MDMMA_i }}\right]
\end{aligned}
$$

where $\rho$ is used to adaptively adjust the weight of the control array unit of online sequential limit learning machine. $0 \leq \rho \leq 1$ adaptive feedback equalization design is realized, and the channel time domain expansion and Doppler expansion are realized [16]. The expansion process is expressed as follows:

$$
\begin{aligned}
& a\left(\theta_{0}+\Delta \theta\right)=\left[1, \exp \left(-j \phi_{1}\right), \ldots, \exp \left(-j(M-1) \phi_{1}\right)\right]^{T} \\
& a\left(\theta_{0}-\Delta \theta\right)=\left[1, \exp \left(-j \phi_{1}\right), \ldots, \exp \left(-j(M-1) \phi_{2}\right)\right]^{T}
\end{aligned}
$$

In order to improve the envelope directivity of data transmission and reduce the output bit error rate (BER), adaptive noise cancellation is adopted and feedback equalization is carried out. The characteristic coefficients of the output envelope are obtained as follows:

$$
b\left(\theta_{0}\right)=\frac{1}{2}\left[a\left(\theta_{0}+\Delta \theta\right)+a\left(\theta_{0}-\Delta \theta\right)\right]
$$

Combined with the pulse response of multipath channel, the relationship between transmission and channel classification selection in online sequential learning machine is obtained as follows:

$$
\begin{aligned}
w_{\mathrm{BLCMV}}= & \tilde{R_{y}^{-1}}\left[a_{t}\left(\theta_{0}\right), b_{t}(\theta)\right] \\
& \times\left(\left[a_{t}\left(\theta_{0}\right), b_{t}(\theta)\right]^{H}\left[\begin{array}{l}
1 \\
g
\end{array}\right]\right.
\end{aligned}
$$

By adopting the channel decision feedback equalization design, the classification and selection function of the online sequential extreme learning machine multi-objective model is obtained as follows:

$$
\begin{aligned}
\tilde{y}(t) & =\int_{-\infty}^{\infty} \tilde{c}(\tau ; t)\left\{\sum_{n=-\infty}^{\infty} \tilde{s}(t-n T) \frac{\sin (2 \pi B(\tau-n T))}{2 \pi B(\tau-n T)}\right\} d \tau \\
= & \sum_{n=-\infty}^{\infty} \tilde{s}(t-n T) \int_{-\infty}^{\infty} \tilde{c}(\tau ; t)\left\{\frac{\sin (2 \pi B(\tau-n T))}{2 \pi B(\tau-n T)}\right\} d \tau \\
& =\sum_{n=-\infty}^{\infty} \tilde{s}(t-n T) \tilde{g}_{n}(t)
\end{aligned}
$$

where the load overhead $W\left(e_{s}\right)$ of online sequential learning machine control channel is described as follows:

$$
W\left(e_{S}\right)=\sum_{v \in e_{s}} S_{N}\left(t^{-}, v\right), \forall v \in e_{s}
$$

Based on the above processing, combining the feedback compensation and the adaptive equalization control method, the classification selection and equalization of the multi-objective model of the ultimate learning machine are realized, and the quality of the multi-objective model of the online sequential ultimate learning machine is improved. 


\section{Experience}

In the experiment, the data transmission rate of online sequential extreme learning machine is $100 \mathrm{kBaud}$. The order of matched filter is 10 , and the iterative step size is 0.023, $a=0.37, \beta=2.5 \times 10^{-5}$, multipath amplitude parameter $(1,0.32,-0.34,0.56,-0.15)$, and multipath delay parameter $(0,0.63,1.63,3.32,3.34)$. The frequency is 3 $\mathrm{kHz}$, the BPSK modulation data information is used as the test signal, and the multi-objective combined control is used to self-adaptively filter the sensing fusion information of the online sequential limit learning machine and to suppress the phase shift and obtain the online sequential limit. The constellation diagram of the multiobjective model of learning machine is shown in Fig. 2.

It can be seen from Fig. 2 that the proposed method is used for adaptive channel selection, which makes the channel equalization assignment performance better and realizes the classification and selection of multi-objective models. Different methods are used for multi-objective classification, and the error code comparison results of the online sequential extreme learning machine are obtained as shown in Fig. 3.

The result of analysis Fig. 3 shows that the method of this paper is used to classify and select the multiobjective model of online sequential extreme learning machine, which reduces the output error and increases the number of ultimate learning machine. Target selection and data transmission quality are improved.

\section{Results and discussion}

In this paper, a multi-objective model selection algorithm is proposed based on feedback compensation and adaptive equalization control. The channel equalization model of online sequential ultimate learning machine is constructed. The sensor fusion information of online sequential limit learning machine is selected adaptively by multiobjective combined control, and the multi-objective combined control is carried out by using matched filtering method. Combined with feedback compensation and adaptive equalization control method, the classification selection and equalization of network multi-objective models are realized. The simulation results show that the algorithm has good accuracy in classifying and selecting multi-objective models of online sequential LLM, and the adaptive equalization performance of channel is better, and the error of LLM control is low. This method has good application value in extreme learning machine control and multi-objective learning.

\section{Abbreviations \\ LLM: Limit learning machine; SLFN: Single-hidden layer feedforward neural network; UHF: Ultra high frequency; VHF: Very high frequency}

\section{Acknowledgements}

This work was supported by Zhejiang Provincial Natural Science Foundation of China under Grant No. LQ15A010009 and LY16G010009, the public welfare science and technology project of Wenzhou city (R20150010), Zhejiang provincial academy of social sciences research (2015 N068), the Science and Technology Project of Wenzhou Science and Technology Bureau No. G20150005, and the Science and Technology Project of Education Department of ZhejiangProvince No. Y201840743.

\section{Funding}

This work was supported by Zhejiang Provincial Natural Science Foundation of China under Grant No. LQ15A010009 and LY16G010009, the public welfare science and technology project of Wenzhou city (R20150010), Zhejiang provincial academy of social sciences research (2015 N068), the Science and Technology Project of Wenzhou Science and Technology Bureau No. G20150005, and the Science and Technology Project of Education Department of ZhejiangProvince No. Y201840743.

\section{Availability of data and materials}

The datasets used and/or analyzed during the current study are available from the corresponding author on reasonable request.

Ethics approval and consent to participate

The research in this paper does not involve any ethical research.

Consent for publication

All authors agree to publish this paper.

Competing interests

The authors declare that they have no competing interests.

\section{Author details}

${ }^{1}$ The First Affiliated Hospital of Wenzhou Medical University, Wenzhou 325000, People's Republic of China. ${ }^{2}$ Oujiang College, Wenzhou University, Wenzhou 325035, People's Republic of China. ${ }^{3}$ Department of Electrical and Electronic Engineering, Wenzhou Vocational \& Technical College, Wenzhou 325035, People's Republic of China. ${ }^{4}$ School of Information and Engineering, Wenzhou Medical University, Wenzhou 325035, People's Republic of China.

Received: 19 January 2019 Accepted: 22 May 2019

Published online: 13 June 2019

\section{References}

1. K. Drakakis, S. Rickard, R.D. Frein, et al., Analysis of financial data using nonnegative matrix factorization. Int Math Forum 3(38), 1853-1870 (2008)

2. B. Ju, Y.T. Qian, M.C. Ye, Collaborative filtering algorithm based on structured projective nonnegative matrix factorization. J Zhejiang Univ Eng Sci 49(7) 1319-1325 (2015)

3. B.F. Li, Y.D. Tang, Z. Han, A geometric structure preserving nonnegative matrix factorization for data representation. Inf. Control. 46(1), 53-59 (2017)

4. Y. Wu, B. Shen, H. Ling, Visual tracking via online nonnegative matrix factorization. IEEE Trans Circuits Syst Video Technol 24(3), 374-383 (2014)

5. M. Ye, Y. Qian, J. Zhou, Multitask sparse nonnegative matrix factorization for joint spectral-spatil hyperspectral imagery denoising. IEEE Trans. Geosci. Remote Sens. 53(5), 2621-2639 (2015)

6. L.R. Hu, J.G. Wu, L. Wang, Application and method for linear projective nonnegative matrix factorization. Comput Sci 40(10), 269-273 (2013)

7. C.H. Ju, J.B. Zou, An incremental classification algorithm for data stream based on information entropy diversity measure. Telecommunications Sci 31(2), 86-96 (2015)

8. Y.X. Lyu, C.Y. Wang, C. Wang, et al., Online classification algorithm for uncertain data stream in big data. J Northeast Univ (Nat Sci Ed) 37(9), 1245-1249 (2016)

9. Y. Chen, L.J. Li, Very fast decision tree classification algorithm based on redblack tree for data stream with continuous attributes. J Nanjing Univ Posts Telecommunications (Nat Sci Ed) 37(2), 86-90 (2017)

10. Z.W. Qiao, W.X. Sun, Two improments to (4.5 algorithm. J Jiangsu Polytechnic Univ 20(4), 56-59 (2008)

11. S.C. Huang, Y. Liu, Classification algorithm for noisy and dynamic data stream. J Jiangsu Univ Sci Technol (Nat Sci Ed) 30(3), 281-285 (2016)

12. B. Sun, J.D. Wang, H.Y. Chen, et al., Diversity measures inensemble learning. Control Decision 29(3), 385-395 (2014)

13. X.P. Hou, X.F. Zhang, X.N. Zhao, Cloud storage performance evaluation research. Comput Sci 41(4), 190-194 (2014) 
14. H.E. Si-Min, K. Mu-Ning, Z. Xiao, et al., Research on Cloud Storage Performance Evaluation Technology and Methods[J]. Comput Mod 12(12), $1-4,8(2011)$

15. C.Y. Qi, Z.H. Li, X. Zhang, et al., The research of cloud storage system performance evaluation. J Comput Res Dev 51(S1), 223-228 (2014)

16. P. Shivam, V. Marupadi, J.S. Chase, et al., Cutting corners:Workbench automation for server benchmarking[C]//Proceedings of USENIX 2008 Annual Technical Conference (USENIX Association, Berkeley, CA, 2008), pp. 241-254

\section{Publisher's Note}

Springer Nature remains neutral with regard to jurisdictional claims in published maps and institutional affiliations.

\section{Submit your manuscript to a SpringerOpen ${ }^{\circ}$ journal and benefit from:}

- Convenient online submission

- Rigorous peer review

- Open access: articles freely available online

- High visibility within the field

- Retaining the copyright to your article

Submit your next manuscript at $\boldsymbol{\sim}$ springeropen.com 